\title{
The Functional Significance of Myocardial Bridges Determinated with 3D Polar Mapping Method
}

\author{
Zsuzsanna Suciu', Alexandra Stănescu², Beáta Jakó \\ ${ }^{1}$ Clinic of Cardiology, University of Medicine and Pharmacy, Tîrgu Mureș, Romania \\ 2 Center of Advanced Research in Multimodality Cardiac Imaging, Cardio Med Medical Center, Tîrgu Mureș, Romania
}

\section{CORRESPONDENCE}

Alexandra Stănescu

76, 22 Decembrie 1989 St 540124

Tîrgu Mureș, Romania

Tel: +40 265217333

Email: alexandrastanescu90@gmail.com

\section{ARTICLE HISTORY}

Received: 16 April, 2016

Accepted: 28 April, 2016
Zsuzsanna Suciu • 38 Gheorghe Marinescu St, 540139 Tîrgu Mureș, Romania, Tel: +40 265215551 Email: szoke_zsuzsanna@yahoo.com

Beáta Jakó • 76, 22 Decembrie 1989 St, 540124 Tîrgu Mureș, Romania, Tel: +40 265217333

Email: jakobeata@yahoo.com
We present the case report of a 39 year-old male patient, without any previous history of cardiovascular events, who was admitted to the Emergency Unit presenting typical angina for 5 hours, associated with diaphoresis and nausea. The physical examination revealed bradycardia ( 57 beats/minute) and a normal blood pressure of 124/67 mmHg. ECG indicated ST-segment elevation of $4 \mathrm{~mm}$ in leads V1-V4 (Panel A). Troponin levels were slightly increased $(0.04 \mathrm{ng} / \mathrm{ml})$, however all the other cardiac enzymes levels were within normal ranges. Due to the high suspicion of acute coronary syndrome, an angiocoronarography was performed, showing no thrombotic lesion and no significant coronary stenosis, however revealing a dynamic stenosis of the proximal segment of the left anterior descending coronary artery (LAD) with a length of $15 \mathrm{~mm}$. For identification of the real cause of myocardial ischemia we performed a 64-Slice Computer Tomography Angiography (MSCT), followed by 3D reconstruction, which illustrated the presence of a myocardial bridge with a length of $20 \mathrm{~mm}$, emerging from the proximal D2 segment of the LAD, with no other stenosis or atherosclerotic plaque (Panel B, C). We evaluated the end-systolic and end-diastolic phase of MSCT images to study the systolic compression in the myocardial bridges. We evaluated three objective parameters to determine the severity of myocardial ischemia, caused by the myocardial bridge, consisting in: the exact surface of ischemic territory, the degree of thickening of the ventricular wall and the amplitude of myocardial segment contraction. Angio CT post-processing allowed the graphical representation of segmental contractility based on color coded polar maps of ventricular contraction. Green color indicates a normal myocardial contraction and blue colors indicate decreased wall motion at the myocardial bridge zone. Polar map analysis identified the correlation between the location of the myocardial bridge and the territory of myocardial ischemia, expressed by an abnormal contraction on angio CT 3D polar map of the ventricular contraction, coded with blue color in the antero-lateral region (Panel D). The patient had a favorable evolution during hospitalization, after initiation 

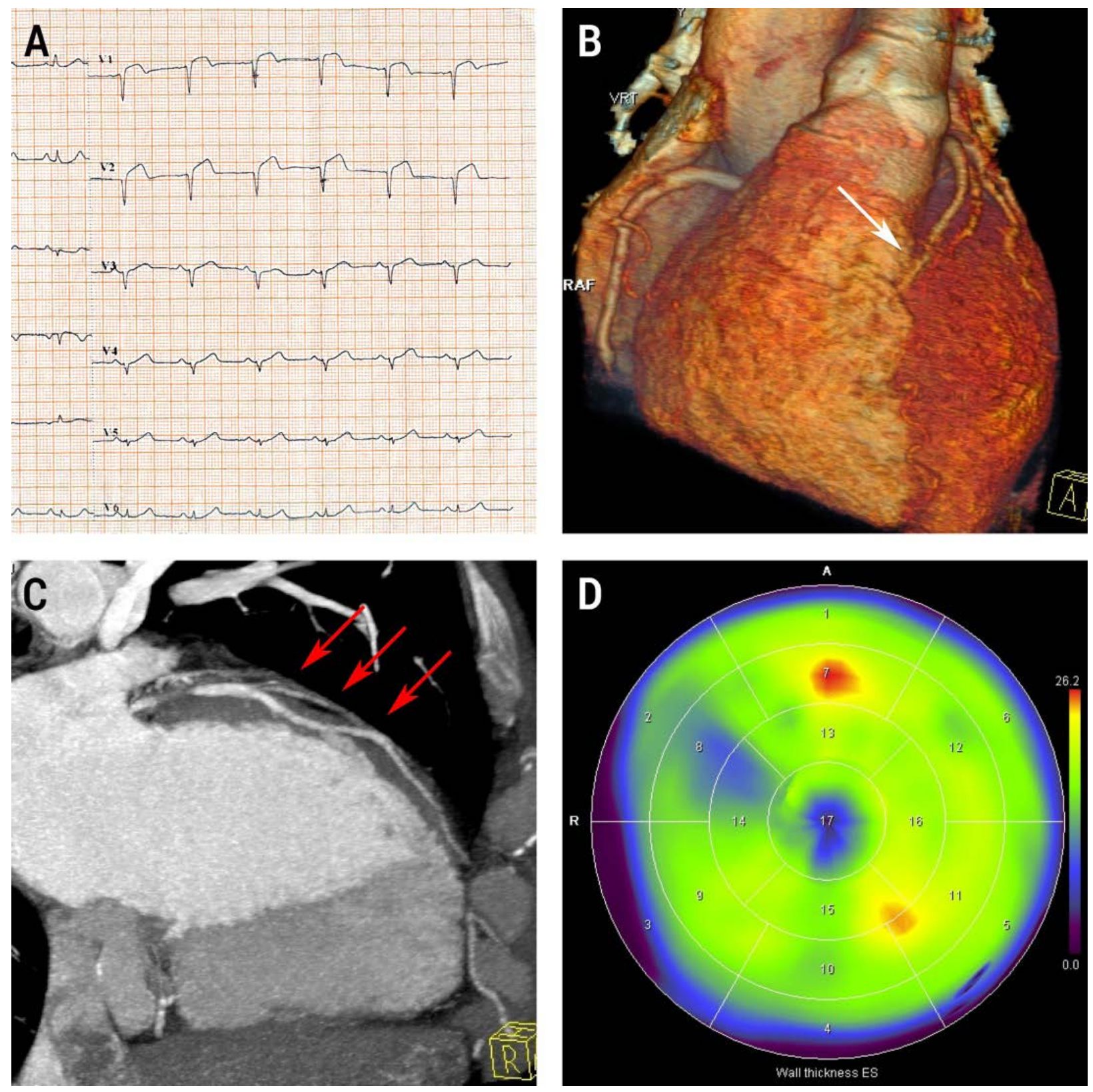

of calcium-channel blockers, without repeating the chest pain. Muscular bridge is a benign, congenital malformation of coronary vessels. Identification of myocardial bridges is a challenging topic for cardiologists, due to its silent appearance, but also due to the severe ischemia that could result from the dynamic obstruction, such as in our case. ${ }^{1-3}$ MSCT allows to perform a complex evaluation of the functional significance of myocardial bridges, detecting the territories with abnormal contractility based on complex 3D polar maps of ventricular contractility, which also shows a

correlation with the severity of the myocardial bridges and their location.

\section{REFERENCES}

1. Erbel R, Ge J, Möhlenkamp S, et al. Myocardial Bridging - A Congenital Variant as an Anatomic Risk Factor for Myocardial Infarction? Circulation. 2009;120:357-359.

2. Ripa Ch, Melatini M , Olivieri F, et al. Myocardial bridging: A 'forgotten' cause of acute coronary syndrome - a case report. Int J Angiol. 2007;16(3):115-118.

3. Abdou M. Myocardial bridging causing ischemia and recurrent chest pain: a case report. Abdou International Archives of Medicine. 2011;4:24. 